\title{
CUATROCIÉNEGAS: CONFLICTOS ASOCIADOS AL TURISMO EN UN ÁREA NATURAL PROTEGIDA
}

\author{
Ivo García Gutiérrez. \\ Álvaro López López. \\ Instituto de Geografía. Universidad Nacional Autónoma de México
}

\section{RESUMEN}

En el Área de Protección de Flora y Fauna de Cuatrociénegas, Coahuila, una de las áreas protegidas con mayor actividad turística en México, se estudiaron entre los años 2008 y 2014 los conflictos derivados de la dinámica turística desde una perspectiva espacial y con base en una metodología de triangulación que combinó la observación participante con entrevistas a actores clave y dos encuestas, una a pobladores locales y otra a visitantes. El estudio evidenció que el desarrollo del turismo en Cuatrociénegas ha ido acompañado de efectos negativos sobre el medio físico, la economía y la dinámica social, los cuales pasan inadvertidos para turistas y pobladores locales. Los primeros, aunque saben que visitan una ANP, no son conscientes de los servicios ambientales que presta a la sociedad en general y de la responsabilidad que tienen de contribuir a protegerla. Por su parte, los segundos afirman terminantemente que el turismo es un factor de desarrollo y pocas veces cuestionan los intereses de quienes se benefician de esta actividad.

Palabras clave: turismo, conservación ambiental, observación participante, Cuatrociénegas.

\section{Cuatrociénegas: tourism-borne conflicts in a Natural Protected Area}

\section{ABSTRACT}

In the Wildlife Protected Area of Cuatrociénegas, Coahuila, one of the most visited protected areas in Mexico, tourism-borne conflicts were analyzed between 2008 and 2014

Recibido: 7 de julio de 2015

Devuelto para su revisión: 21 de diciembre de 2015

Aceptado: 26 de febrero de 2016

Departamento de Geografía Económica. Instituto de Geografía. Universidad Nacional Autónoma de México. Dirección: Circuito Exterior s/n, Ciudad Universitaria, Distrito Federal, C.P. 04510 MÉXICO. E-mail: ivo_garcia@hotmail.com; lopuslopez@yahoo.com. 
from a spatial perspective, combining participant observation with surveys of key players, both locals and visitors. The study confirmed the negative effects of tourism on the physical environment as well as on the local economy and social dynamics, a fact that seems to go unnoticed by locals as well as tourists. Although they are aware that they have entered an NPA, tourists are indifferent to the responsibility their visit entails and ignorant of the environmental services the fragile ecosystem provides to society in general. For their part, local residents view tourism as a road to development, and rarely question the interests of those who benefit from the activity.

Keywords: Tourism, environmental protection, participant observation, Cuatrociénegas.

\section{INTRODUCCIÓN}

El turismo se considera hoy una alternativa para promover el desarrollo nacional, regional y local, especialmente en los países menos desarrollados (Watson, et al., 2014; Arsuaga y Jiménez, 2014). Su capacidad de contribuir a reducir la pobreza proviene de la cadena de valor que genera a través de sus múltiples conexiones con otros sectores de la economía, tales como la agricultura, la construcción, los servicios públicos y el transporte (Monterrubio et al., 2013; OIT, 2011). En esa misma lógica, se da por hecho que el arribo de visitantes a las áreas naturales protegidas (ANP) de México ha generado beneficios económicos.

En México la actividad turística es vista como una manera de fomentar el desarrollo económico (Solari y Pérez, 2005; Meixueiro, 2008). En 2013 el turismo representaba 8.7 por ciento del Producto Interno Bruto, lo que lo posicionó como uno de los ejes para el desarrollo del país (INEGI, 2015; Sistema Integral de Información de Mercados Turísticos, 2015). Aunque menor, una parte del sector turístico se ubica en las ANP (Susana, 2005; Sectur, 2007; Brenner, 2010), las cuales se han convertido en instrumentos de política pública para promover el desarrollo económico, el aumento de la calidad de vida de sus habitantes y la salvaguarda de los recursos naturales (Watson, et al., 2014; Maekawa, et al., 2013; Pérez, et al., 2010).

En los países subdesarrollados, las ANP se establecen en terrenos con población, por lo que cumplen además una función social (Roe, et al, 2003; De Oliveira, 2002). En el caso de México, una tercera parte de las ANP (creadas por decreto federal, estatal o municipal) cuenta con población residente, y la mayoría de sus habitantes (90 por ciento) enfrenta algún grado de marginación social (Conabio, 2009; Conanp, 2014). Por ello el turismo se promueve como una actividad alternativa para favorecer a la población local (Conanp, 2007a). Las cifras más recientes estiman que alrededor de 14 millones de personas visitaron las ANP de México durante el año 2007, lo cual generó una derrama económica por la prestación directa del servicio turístico de unos 8,345 millones de pesos y más de 27 mil empleos (Bezaury-Creel, 2009; Conanp, 2007a).

Sin embargo, el turismo puede tener impactos negativos cuando se realiza sin planeación y organización adecuadas, y cuando no se consideran los actores locales (Andereck et al, 2005; Tinoco, 2003; Meixueiro, 2008). La amenaza de los impactos negativos del turismo se agrava al interior de las ANP porque en ellas convergen zonas de marginación social y ecosistemas únicos de alta fragilidad (Conanp, 2007a). 
En el caso de Cuatrociénegas, dos acontecimientos detonaron su arranque como destino turístico: el decreto federal de 1994 del Valle de Cuatrociénegas como ANP, y el que en 1995 George Grall publicara en la revista National Geographic un artículo sobre Cuatrociénegas, lo que posicionó al valle como destino turístico de alcance internacional (Grall, 1995; Núñez, 2006; Galván, 2007). Así, en los últimos 15 años, Cuatrociénegas pasó de recibir apenas un centenar de visitantes anuales durante los periodos vacacionales a acoger 18,503 en 2008 (Conanp, 2008a, 2008b), con una derrama económica estimada de 29.2 millones de pesos (Conanp, 2008c).

Sin embargo, con el incremento de la actividad turística también aumentaron sus efectos negativos, lo cual llevó a la Procuraduría Federal de Protección al Ambiente (Profepa) a clausurar dos sitios de uso público en los años 2004 y 2008, con una serie de conflictos entre los prestadores de servicios turísticos, la población local y las dependencias de gobierno (Núñez, 2006).

Este trabajo se propone reflexionar sobre los efectos espaciales y los conflictos sociales que han acompañado al desarrollo del turismo en el Área de Protección de Flora y Fauna de Cuatrociénegas, para lo cual se divide en cuatro apartados: en el primero, denominado “Área de Protección de Flora y Fauna Cuatrociénegas", se expone la localización, ubicación y descripción básica del espacio de estudio; en el segundo, "Metodología", se explica que se triangularon metodologías cuantitativas (encuestas a turistas y habitantes locales) con cualitativas (observación participante y entrevistas) que permitieron contextualizar y revelar los conflictos derivados del turismo; en el tercero, "Contexto básico de los turistas y los residentes" se expone el perfil de los actores involucrados en el fenómeno turístico, tanto visitantes como pobladores locales, y en el cuarto apartado, "El turismo en el ANP Cuatrociénegas: entre conflictos y posibles soluciones", se exponen en primer lugar las problemáticas identificadas a través de la observación participante $\mathrm{y}$, posteriormente, se interpretan sus causas y se sugieren algunas propuestas que podrían ofrecer alternativas de solución.

\section{2. ÁREA DE PROTECCIÓN DE FLORA Y FAUNA DE CUATROCIÉNEGAS}

El Área de Protección de Flora y Fauna de Cuatrociénegas se localiza en el municipio del mismo nombre, en la porción central del estado de Coahuila, México, en el corazón del Desierto Chihuahuense (Figura 1). Fue decretada el 7 de noviembre de 1994 y abarca una superficie de 84,347 hectáreas, con paisajes y ecosistemas únicos y contrastantes: matorrales desérticos, pastizales, dunas y humedales (INE, 1999; Secretaría de Gobernación, 1994). Su administración está a cargo de la Comisión Nacional de Áreas Naturales Protegidas (Conanp).

El clima dominante en la región es semicálido muy seco, con lluvias en verano, invierno fresco y muy bajo porcentaje de lluvias invernales (García, 1987). La temperatura varía de $0^{\circ} \mathrm{C}$ en invierno hasta $40^{\circ} \mathrm{C}$ en verano, con una media anual de $21.2^{\circ} \mathrm{C}$. La media mensual más alta llega a rebasar $\operatorname{los} 30^{\circ} \mathrm{C}$ en los meses de junio, julio y agosto. La precipitación media anual es de $221 \mathrm{~mm}$ en el valle, y la evaporación potencial de más de $2 \mathrm{mil} \mathrm{mm}$. Se ha estimado que en el valle de Cuatrociénegas este clima ha existido por más de 30 mil años (Meyer, 1973; Salas et al., 2010; Secretaría de Gobernación, 2013). 


\section{Figura 1 \\ UBICACIÓN GEOGRÁFICA DEL ANP CUATROCIÉNEGAS}

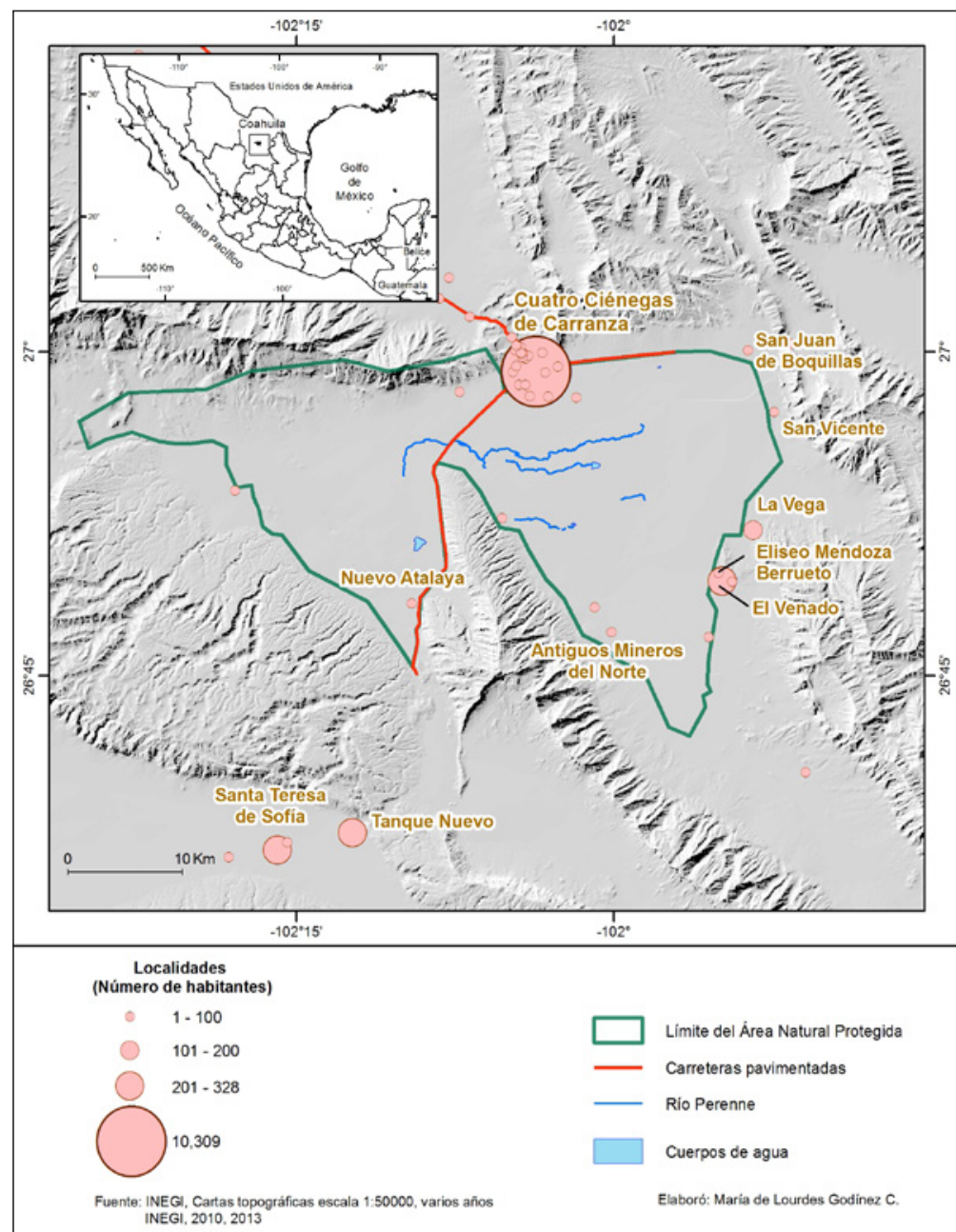

Fuente: elaborado por María de Lourdes Godínez Calderón, 2015, con base en INEGI (2010 y 2013).

En las partes más bajas del valle se desarrolla vegetación halófila (pastizales), aunque también se encuentran áreas desprovistas de vegetación, como las dunas, y otras de vegetación subacuática, como los humedales. Los matorrales se encuentran en zonas ligeramente más elevadas, de los cuales el submontano es el de mayor altitud (INE, 1999). Cuatrociénegas es un sitio de alta biodiversidad, con más de 70 especies endémicas de humedal, entre ellas peces, caracoles y bacterias. El sitio alberga 1,247 especies de fauna, 16 de las cuales están en peligro de extinción, 39 amenazadas y 34 bajo protección especial (Souza, et al., 2006; Escalante, et al., 2008; Lobo 2009). 
En el valle de Cuatrociénegas existen aproximadamente 500 cuerpos de agua superficiales conocidos localmente como pozas o ciénegas, los cuales brotan de manantiales con diámetros que van desde menos de un metro hasta más de cien, y profundidades de entre 50 centímetros y 18 metros. Algunas de las pozas se comunican natural o artificialmente entre sí mediante un complicado sistema de drenaje (INE, 1999; Conagua, 2009; Secretaría de Gobernación, 2008).

La superficie de este humedal se ha reducido a 251 hectáreas en los últimos años debido a la sobreexplotación del recurso hídrico con fines predominantemente agrícolas. De los 99.5 millones de metros cúbicos de agua que brota anualmente de las pozas, más de tres cuartas partes se extraen de la cuenca para el riego de parcelas agrícolas fuera del municipio de Cuatrociénegas. También existe sobreexplotación de agua subterránea, por lo que en 2013 se impuso una veda al acuífero (Conanp, 2007b; Conagua, 2009; Secretaría de Gobernación, 2013).

En el municipio de Cuatrociénegas viven 13,013 personas, y su densidad de población es de 1.22 habitantes por kilómetro cuadrado, lo cual contrasta con los 18.24 habitantes por kilómetro cuadrado del estado de Coahuila, uno de los de menor densidad demográfica en México. La población masculina representa 50.44 por ciento, lo cual resulta en una proporción de género bastante equilibrada. El municipio cuenta con 96 localidades de dos o más viviendas, y el 95 por ciento de la población se concentra en la cabecera municipal de Cuatrociénegas (Figura 1; INEGI, 2011).

El municipio de Cuatrociénegas se encuentra seis puntos por encima de la media estatal y 11 por encima de la media nacional en el rubro de población vulnerable por bajos ingresos. Entre los problemas más acuciantes destacan: a) la presencia de 5,090 personas en situación de pobreza (43.7 por ciento de la población total); b) 6,993 habitantes que perciben un ingreso inferior a la línea de bienestar (60.1 por ciento de la población total); c) 2,761 personas en la línea de bienestar mínimo (23.7 por ciento de la población total), y d) más de la mitad de la población (7,438 personas, 63.9 por ciento del total) tienen al menos una carencia social (INEGI, 2011; Inapac, 2014).

Como se puede apreciar en la Figura 1, hay 10 localidades entre uno y cien habitantes, de las cuales Antiguos Mineros del Norte es la más numerosa, con 97. La densidad de población del ANP es apenas de 0.11 habitantes por kilómetro cuadrado, y fuera de ella se ubican las localidades más numerosas, siendo la cabecera municipal de Cuatrociénegas, con más de 10 mil habitantes, la más importante en términos históricos, económicos, sociales y políticos. Otras localidades de importancia regional son La Vega y El Venado (colindantes con el ANP) y, aunque más alejadas, están también Santa Teresa de Sofía y Tanque Nuevo (INEGI, 2011).

La población económicamente activa en la localidad de Cuatrociénegas de Carranza es de 2,825 personas (31.72 por ciento de la población total), empleadas en los siguientes sectores: 314 (11.60 por ciento) en el sector primario (agricultura, explotación forestal, ganadería, minería, pesca); 879 (32.47 por ciento) en el sector secundario (construcción, electricidad, gas y agua, industria manufacturera) y 1,514 (55.93 por ciento) en el sector terciario (comercio, servicios y transportes). 
La clasificación en el municipio es de 42.74 por ciento en el sector primario, 22.61 por ciento en el sector secundario y 34.66 por ciento en el terciario, mientras que en el estado las cifras son 50.82 por ciento, 5.47 por ciento y 43.71 por ciento, respectivamente.

Antes del decreto del ANP en 1994 no había ningún control de acceso a las pozas del humedal; cualquier persona podía llegar a ellas a pie, a caballo, en automóvil o en bicicleta, sin necesidad de pedir permiso a los dueños de los predios o a los ejidatarios, y sin cubrir cuotas de acceso. Al inicio de los años setenta del siglo XX dos balnearios en el humedal abrieron sus puertas al público: Río Mezquites, actualmente en operación, propiedad de la familia Arredondo-Galindo, y Poza de la Becerra (clausurado al turismo en 2008). Los dueños de estos sitios construyeron en un principio palapas y bancas de madera para recibir a sus familiares y amigos, y con el tiempo comenzaron a recibir turistas.

Aunque el ANP se decretó en 1994, fue hasta 1997 cuando el gobierno federal contrató la primera plantilla de personal para operar el sitio. La entonces presidente municipal de Cuatrociénegas por el Partido Revolucionario Institucional (1994-1996) pasó a ser directora del ANP durante 10 años (1997-2007) y al mismo tiempo empresaria hotelera local. Esto es importante pues, como se verá más adelante, la visión empresarial en el manejo de los recursos naturales se ha contrapuesto a las necesidades de conservación del ANP.

En 1999 se publicó el Programa de Manejo del ANP en el que se incluyeron las primeras normas de uso turístico del sitio, tales como la prohibición de cuatrimotos y el registro y autorización previos de los visitantes. Fue entonces que comenzaron a ordenarse las actividades turísticas y recreativas en el valle de Cuatrociénegas (INE, 1999; Galván, 2007).

\section{METODOLOGÍA}

La metodología de la investigación combinó métodos cuantitativos y cualitativos (Decrop, 2004; Arias, 2009; Yeasmin y Rahman, 2012). La investigación cualitativa (Martin, 2010; Phillmore y Goodson, 2004) se dio en un estudio longitudinal desde la perspectiva del "observador participante" (Cole, 2005), tomando en cuenta la posición y experiencia personal del primer autor de este trabajo, residente de Cuatrociénegas, en calidad de ciudadano, investigador y funcionario de la Conanp como Director del Área de Protección de Flora y Fauna de Cuatrociénegas (del 5 de febrero de 2008 al 4 de noviembre de 2012).

Entre 2008 y 2012 se aplicaron además técnicas cualitativas para la investigación de la población local (Cole, 2007; Brenner, 2010; López et al., 2010; Monterrubio et al., 2011; Brenner y Job, 2006), tales como entrevistas, sesiones, participaciones en reuniones, talleres y mesas de trabajo; todas ellas relacionadas con el turismo del ANP de Cuatrociénegas y con la participación de empresarios del sector turístico, prestadores de servicios turísticos, funcionarios de los tres niveles de gobierno y organizaciones civiles, así como público en general, incluyendo turistas y residentes locales.

A fin de complementar la investigación se aplicaron dos encuestas, una a la población local y otra a los turistas. La importancia de la encuesta para conocer la percepción de los actores locales ha sido ampliamente establecida (Andriotis y Vaughan, 2003; Jurowski y Gursoy, 2004; Wang y Pfister, 2006; Nunkoo y Gursoy, 2012, Casals, y Toure, 2013). En este caso interesaba conocer la percepción y actitudes que del turismo tiene la población local. Entre marzo y julio de 2012 se encuestó aleatoriamente a 374 residentes locales del 
municipio de Cuatrociénegas, personas mayores de 18 años con más de un año de residencia, pertenecientes a todos los sectores de la población. La proporción de género de los encuestados fue de 239 mujeres (64 por ciento) y 135 hombres (36 por ciento). Se utilizó la técnica de análisis factorial para eliminar la información redundante (Brida et al., 2012).

Por su parte, las encuestas a turistas es una práctica cada vez más común entre quienes se interesan por el fenómeno del turismo en espacios de alta fragilidad (Brenner y Job, 2006; García, et al., 2006; Márquez y Sánchez, 2007). La encuesta a los visitantes a Cuatrociénegas se levantó entre 2008 y 2013. Se aplicaron 1,260 cuestionarios en sitios de uso público, concretamente en el balneario Río Mezquites (Figura 1), para conocer su procedencia, su perfil socioeconómico, su conocimiento del sitio, su nivel de satisfacción con los servicios prestados y sus actitudes hacia los impactos del turismo.

Ambas encuestas se realizaron con ayuda del personal de la Conanp, estudiantes y voluntarios, principalmente del Centro de Bachillerato Tecnológico 22 de Cuatrociénegas, Coahuila, de la Universidad Tecnológica del Valle del Mezquital y del Instituto de Geografía de la Universidad Nacional Autónoma de México.

\section{CONTEXTO BÁSICO DE LOS TURISTAS Y LOS RESIDENTES}

\subsection{Los turistas}

Casi la totalidad (98 por ciento) de los visitantes encuestados en el Área de Protección de Flora y Fauna de Cuatrociénegas era de origen nacional, y sólo dos por ciento provenía de otros países, como Estados Unidos, Costa Rica, Canadá, Guatemala y Colombia. Los turistas nacionales eran principalmente de Coahuila (86 por ciento) y estados vecinos: seis por ciento de Nuevo León, tres por ciento de Chihuahua y uno por ciento del estado de Durango, y el resto de otros estados, como Guanajuato (dos por ciento) y Distrito Federal (dos por ciento).

Los visitantes de Coahuila provenían principalmente de los municipios de Monclova (54 por ciento), Cuatrociénegas (12 por ciento), Saltillo (ocho por ciento), Frontera (cinco por ciento), Castaños (cinco por ciento), San Buenaventura (cuatro por ciento), Torreón (tres por ciento) y Ocampo (dos por ciento). En cuanto a la proporción de género, 51 por ciento eran hombres y 49 por ciento mujeres. La mayoría (37 por ciento) tenía entre 21 y 30 años de edad, seguida por el intervalo de catorce a veinte años (diecinueve por ciento), siete a trece años (trece por ciento) y 31 a 40 años (doce por ciento). Los menores de 13 años representaron 22 por ciento de los visitantes.

Por otra parte, 80 por ciento de los visitantes del ANP ya había visitado anteriormente el lugar (5.4 visitas en promedio), y para 20 por ciento de ellos era su primera visita. Los visitantes permanecieron en promedio 2.6 días en Cuatrociénegas. Los motivos más significativos para visitar el lugar fueron: vacaciones (74 por ciento), visita a familiares en Cuatrociénegas (25 por ciento) y negocios (uno por ciento). Durante sus visitas, 59 por ciento buscaba relajación, 30 por ciento nadar, 22 por ciento disfrutar de los paisajes, 13 por ciento disfrutar del clima, 11 por ciento aprender de la naturaleza y 26 por ciento no tenía una preferencia específica. La mayoría de los turistas encuestados (90 por ciento) hubiera deseado poder acampar en el ANP, lo cual hasta ahora no es posible, pero puede considerarse un área de oportunidad. 
El gasto promedio de los turistas en el ANP fue de 244 pesos mexicanos (19.5 dólares estadounidenses). Los turistas gastaban mayormente en alimentos, alojamiento y transporte. Antes de llegar al ANP adquirieron sus víveres en Cuatrociénegas (50 por ciento), Monclova (34 por ciento), Comarca Lagunera (cinco por ciento), Monterrey (dos por ciento) y otros lugares no especificados (nueve por ciento). Aunque la gran mayoría de los turistas (90 por ciento) sabía que visitaba un ANP, poco entendían acerca de la importancia de sus servicios ambientales y de su conservación: 63.70 por ciento lo desconocía totalmente (a juzgar por sus respuestas inconexas o por haber dejado los espacios en blanco); 32.90 por ciento sabía poco (respuestas con alguna noción de lo que es un ANP y la mención de por lo menos uno de sus objetos de conservación) y sólo 3.40 por ciento tenía un conocimiento significativo (respuestas con una buena definición de lo que es un ANP y la identificación de sus objetos de conservación y sus beneficios ambientales) (Figura 2).

\section{Figura 2}

\section{NIVEL DE CONOCIMIENTO DE LOS TURISTAS Y LOS POBLADORES LOCALES. ACERCA DEL ANP Y SUS OBJETOS DE CONSERVACIÓN (EN PORCENTAJE)}

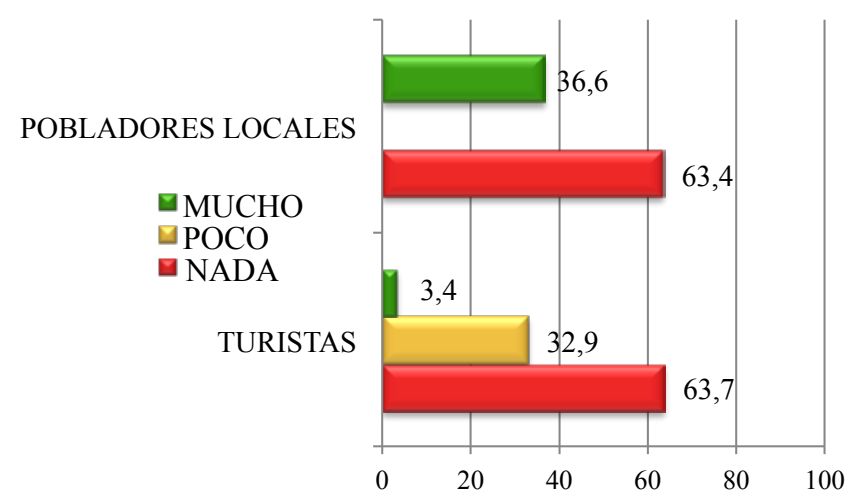

Fuente: elaboración propia con base en datos de campo e información de Conanp (2012).

En cuanto al nivel de satisfacción, 80 por ciento lo reportó como bueno, 18 por ciento como regular y uno por ciento como malo. Los turistas esperaban encontrar otros servicios, tales como visitas guiadas, pláticas informativas y folletos. Casi la mitad (45 por ciento) de ellos consideró que el trato recibido durante su estancia fue bueno, 26 por ciento que fue regular, 23 por ciento excelente y 16 por ciento que fue malo. Respecto al impacto del turismo sobre el medio natural, 61 por ciento considera que el turismo no afecta al medio natural, mientras que el 39 por ciento que lo afecta negativamente. De esta parte, 32 por ciento considera que los turistas locales son quienes más ocasionan daños al medio natural, 36 por ciento que son todos los turistas, 19 por ciento que son los extranjeros y 13 por ciento culpa a los nacionales. En cuanto a los beneficios del turismo para las comunidades rurales vecinas, 67 por ciento de los turistas considera que se ven beneficiadas por la actividad turística, y 33 por ciento opina lo contrario. 


\subsection{Los pobladores locales}

Seis de cada 10 cieneguenses consideran que el turismo fortalece y diversifica la economía local, y siete de cada diez que el turismo promueve la creación de tiendas y restaurantes entre los pobladores locales. Sin embargo, con la llegada de las grandes franquicias, como Oxxo (tiendas de conveniencia) en 2011 y Soriana (supermercado) en 2013, los inversionistas foráneos han empezado a integrarse al mercado de Cuatrociénegas, lo que ha conducido a cambios en la dinámica económica local desfavorables al desarrollo de capitales internos.

Otra noción dominante entre los residentes locales (58 por ciento) es que el turismo en Cuatrociénegas no afecta la conservación de las áreas naturales. Esto puede relacionarse con su desconocimiento del medio natural, tomando en cuenta que, de acuerdo con los resultados de las evaluaciones realizadas por el personal del ANP (Conanp, 2012), sólo 36.6 por ciento de los cieneguenses fue capaz de identificar los objetos de conservación del área natural protegida y las situaciones o factores que amenazan su conservación (Figura 2). Por otra parte, 51 por ciento de los encuestados considera que el turismo en Cuatrociénegas no afecta la conservación de sitios histórico-culturales, como cuevas con pinturas rupestres. No obstante, tres de cada 10 consideran que el turismo genera crimen y vandalismo en sus comunidades.

Asimismo, 25 por ciento de la población local considera que el turismo en Cuatrociénegas afecta la calidad de sus oportunidades recreativas. Cinco de cada 10 declaró no visitar lo sitios de uso público en el ANP por diversas razones: alto costo de las cuotas de acceso (28.5 por ciento), no les gustan los sitios (23.36 por ciento), no cuentan con recursos económicos para hacerlo (9.35 por ciento), creen que está prohibido entrar (7.94 por ciento), no disponen de tiempo suficiente para la recreación (7.48 por ciento), reciben maltrato del personal del ANP (6.54 por ciento), no cuentan con los medios de transporte para realizar la visita (5.14 por ciento), sus obligaciones de trabajo no se lo permiten (3.27 por ciento), consideran que está prohibido nadar (2.80 por ciento), por la mala calidad en la infraestructura de los sitios de uso público (2.34 por ciento), por la distancia $(<1.00$ por ciento), la edad avanzada ( $<1.00$ por ciento) la aglomeración de personas $(<1.00$ por ciento), por contar con una alberca en sus domicilios $(<1.00$ por ciento), o por motivos religiosos $(<1.00$ por ciento)

Finalmente, 47 por ciento de los cieneguenses considera que el turismo contribuye a mejorar la calidad de la educación. Sin embargo, de acuerdo con el Inapac (2014), 13.85 por ciento de ellos se encuentra en rezago educativo, y 44.3 por ciento no ha terminado la educación básica.

\section{EL TURISMO EN EL ANP DE CUATROCIÉNEGAS: ENTRE CONFLICTOS Y POSIBLES SOLUCIONES}

\subsection{Conflictos derivados del turismo}

Con la llegada de más visitantes nacionales y extranjeros atraídos por los humedales de Cuatrociénegas, los comerciantes y empresarios locales comenzaron a ver al turismo como una alternativa para su desarrollo, y el aumento de los servicios turísticos se hizo 
evidente. Al inicio de la década de 1990 existían en el Valle de Cuatrociénegas dos hoteles, dos restaurantes y dos balnearios; en la actualidad existen 12 hoteles, 15 restaurantes, 12 sitios de uso público y 308 empleados en el sector turístico alrededor del ANP (Comité Pueblo Mágico, 2011).

En 1998 el Centro de Información Poza Azul (Figura 3) abrió sus puertas al público en el corazón del ANP con un enfoque educativo y contemplativo. En 2001 se abrió al público con fines turísticos el sitio Dunas de Yeso (Figura 3), clausurado como yacimiento de explotación minera en 1996; medida que, a decir de Galván (2007), provocó uno de los primeros conflictos sociales derivados de las acciones gubernamentales de protección del sitio.

Figura 3

SITIOS DE INTERÉS TURÍSTICO Y/O DE CONFLICTO EN EL ANP DE CUATROCIÉNEGAS

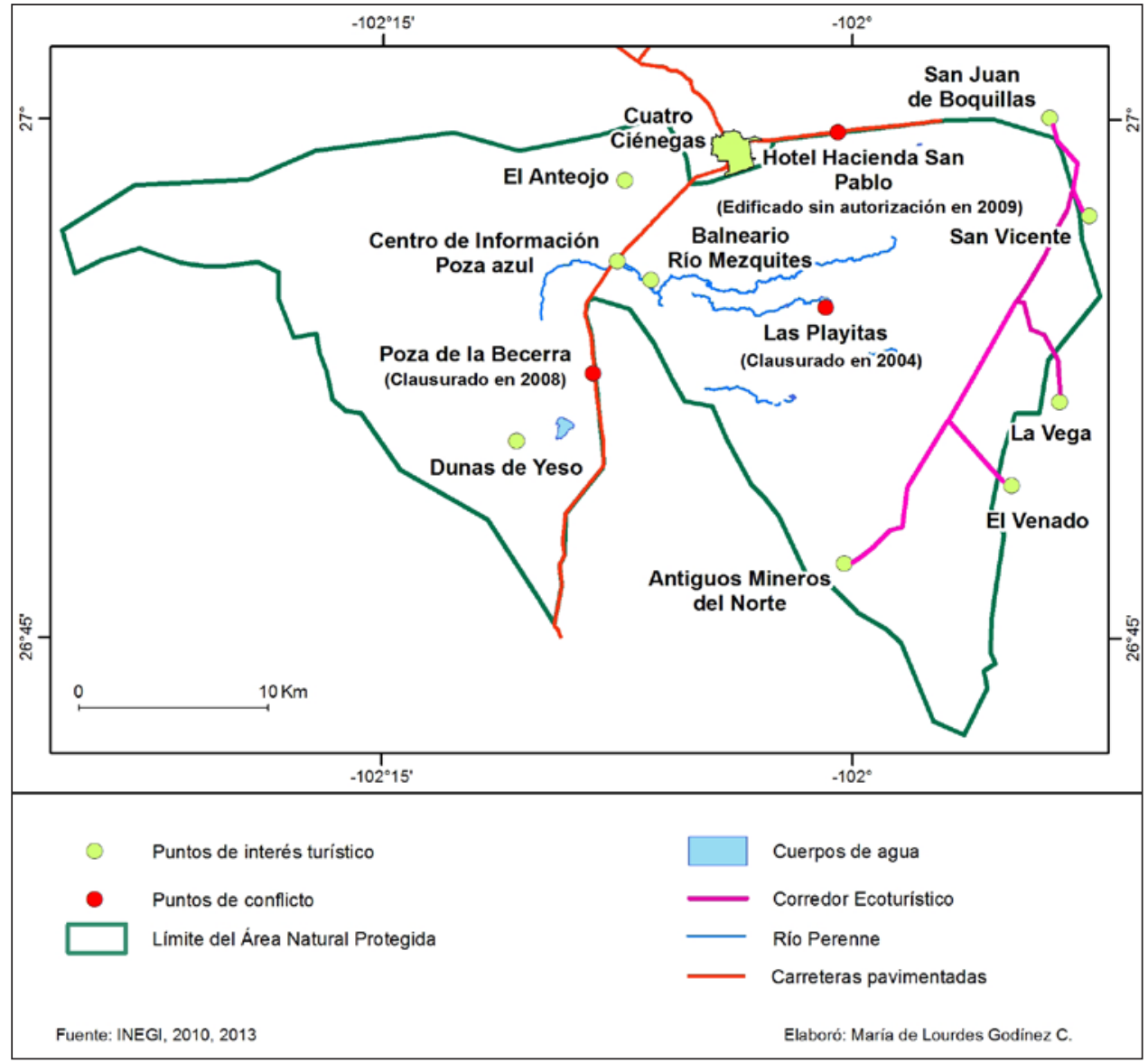

Fuente: elaborado por María de Lourdes Godínez Calderón, 2016, con base en INEGI (2010 y 2013). 
En 2002 la Dirección del ANP instituyó la idea de un "corredor ecoturístico" (Figura 3) que incluiría, con apoyo de programas subsidiarios de la Conanp, infraestructura rústica en las distintas comunidades rurales del corredor: palapas de madera y carrizo, albercas, asadores de carne y señalización básica. Pero los balnearios funcionaron únicamente durante los períodos de alta afluencia; algunos cerraron totalmente tras un par de años, como el caso del ejido Nueva Atalaya (Figura 1 y 3). Los grupos conformados por el personal del ANP para administrarlos pronto se disolvieron por la desorganización en las comunidades. Es decir, los balnearios no lograron convertirse en un negocio redituable para la comunidad y los pobladores perdieron el interés en ellos y dejaron de participar. En la actualidad, el mantenimiento y operatividad de la infraestructura del Corredor Ecoturístico depende de los subsidios de la Conanp y de otras dependencias gubernamentales, además de que su funcionamiento no está vinculado con los servicios turísticos ofertados en la ciudad de Cuatrociénegas, lo cual ha incidido en que ni los hoteleros ni los guías de turistas de la cabecera municipal incluyen al Corredor Ecoturístico dentro de los servicios que ofrecen.

En 2003 la Dirección del ANP inició una masiva campaña promocional para posicionar a Cuatrociénegas como destino turístico. La Secretaría de Turismo de Coahuila y el municipio de Cuatrociénegas hicieron su parte. Como resultado de la intensa promoción, en la Semana Santa de 2004 más de 20 mil personas llegaron a Cuatrociénegas, entonces una localidad de menos de nueve mil habitantes (Figura 4).

\section{Figura 4 \\ LAS PLAYITAS DURANTE LA SEMANA SANTA DE 2004}

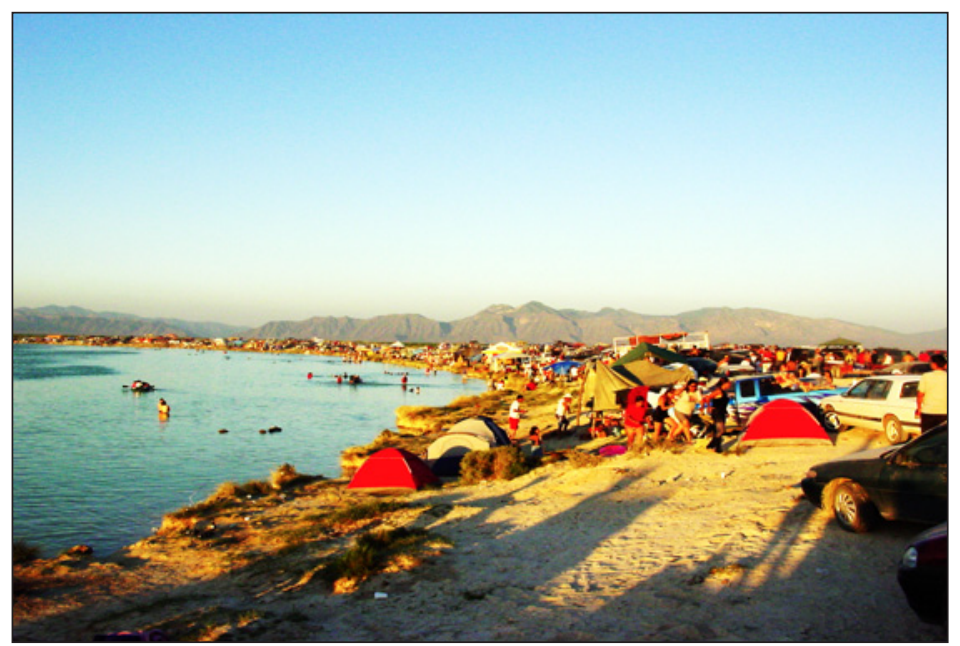

Fuente: Archivo Conanp.

Los balnearios del Corredor Ecoturístico se saturaron, al igual que ocurrió con los de Río Mezquites y Poza de la Becerra, al interior del ANP. Los turistas fueron conducidos entonces a Las Playitas, un humedal en terrenos de litigio. El sitio se convirtió en un caos 
por la aglomeración: había motocicletas y vehículos que abrían caminos por doquier, con las consiguientes pérdida de vegetación, erosión y afectación de la fauna. Nadie se responsabilizó del manejo de los visitantes en el humedal, ni de sus desechos. En un solo día el personal del ANP llenó de basura 11 camionetas pick up. La Procuraduría Federal para la Protección del Ambiente (Profepa) clausuró Las Playitas después de la Semana Santa de 2004 (Figuras 3 y 4).

La visita de decenas de miles de personas durante 2004 provocó que algunas familias pudientes locales de agricultores y ganaderos, comerciantes y de la política incursionaran en el sector turístico. Se construyeron entonces los hoteles Misión Mariaelena, Quinta Santa Cecilia, Posada del Valle y Hacienda San Pablo.

Durante la Semana Santa de 2008 Cuatrociénegas recibió 18,503 turistas. Los balnearios se saturaron, especialmente Poza de la Becerra, donde se presentaron problemas de contaminación, aglomeración y desmonte de vegetación (Figuras 3 y 5). La Profepa clausuró el balneario ese mismo año por motivos similares a la clausura de Las Playitas en 2004 , lo cual ocasionó varias protestas por parte de los pobladores locales, quienes veían amenazadas sus inversiones en el turismo del ANP (Figura 6).

\section{Figura 5 \\ POZA DE LA BECERRA EN LA SEMANA SANTA DE 2008}

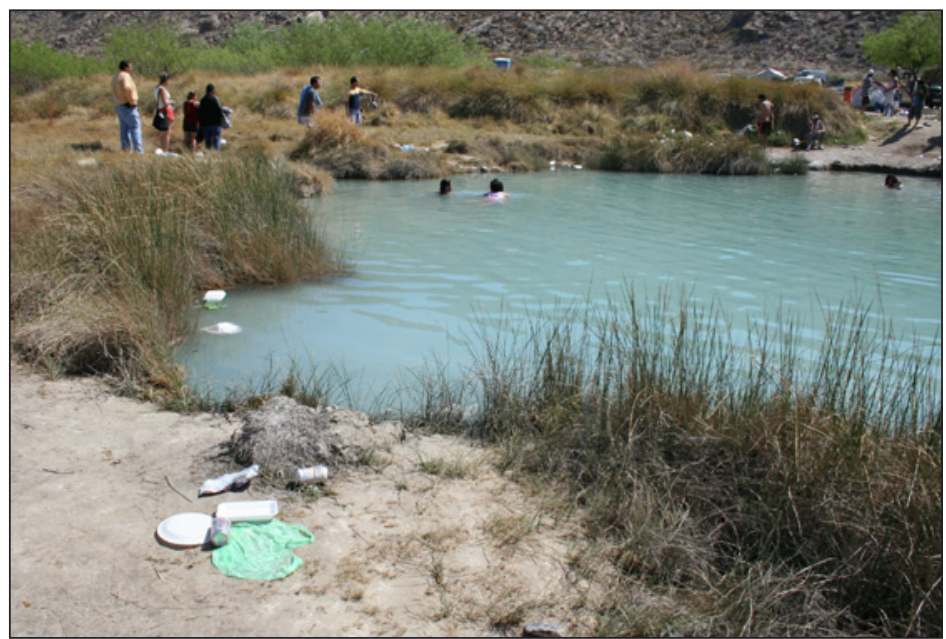

Fuente: Archivo Conanp.

El ordenamiento de la actividad turística generó además otros conflictos (Figuras 3 y 5). A partir de 2008 el personal de la Conanp implementó operativos especiales para limitar el acceso de visitantes a sitios no autorizados, especialmente durante Semana Santa y los fines de semana largos, para evitar aglomeraciones e impactos en los humedales. Esto generó enojo y descontento entre los pobladores locales, quienes vieron limitadas sus oportunidades recreativas. 


\section{Figura 6 \\ POBLADORES LOCALES INCONFORMES CON LA CLAUSURA DE LOS BALNEARIOS}

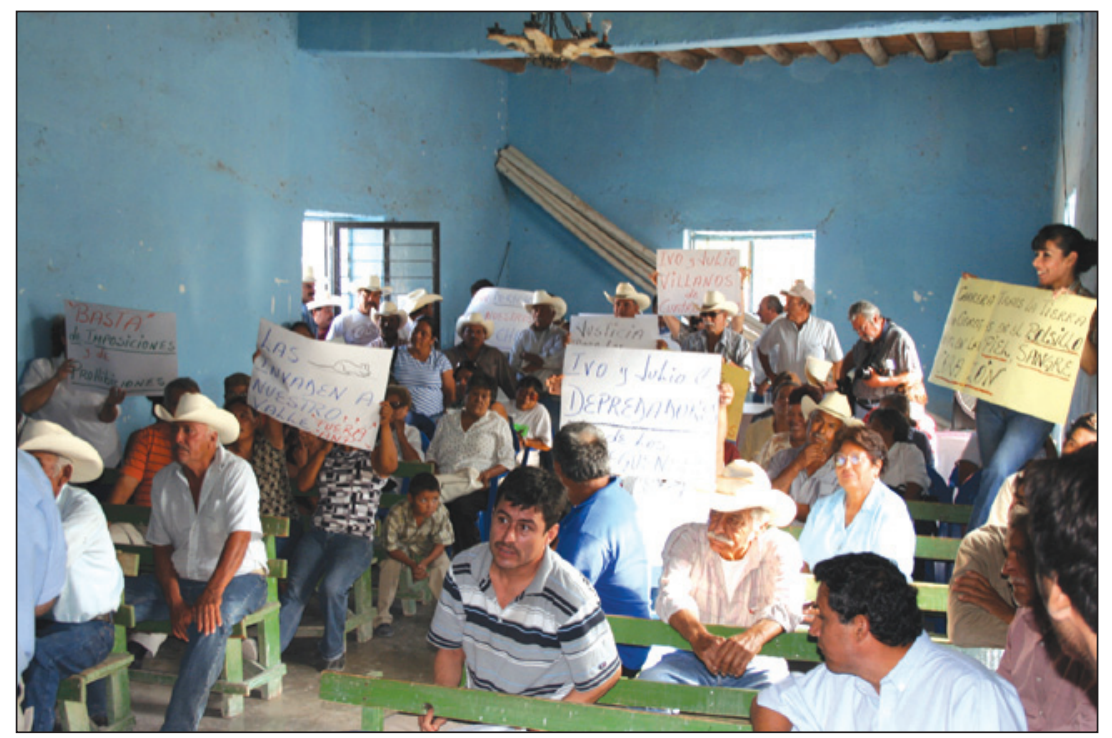

Fuente: Archivo Conanp.

Es claro que el desarrollo turístico en el sitio ha estado marcado por la influencia de la clase política cieneguense. Sobresale el caso del Hotel Hacienda San Pablo (Figura 3), construido por un empresario local al interior del ANP sin contar con las autorizaciones necesarias en materia de impacto ambiental y de cambio de uso de suelo y que fue inaugurado durante la Semana Santa de 2009. A pesar de las múltiples denuncias ante la Profepa, en la actualidad el hotel continúa abierto al público, gozando de total impunidad.

En 2012 la ciudad de Cuatrociénegas recibió la designación de Pueblo Mágico ${ }^{1}$ por parte de la Secretaría de Turismo, lo cual detonó diversas acciones e inversiones para contribuir al desarrollo turístico de la localidad y contribuyó a mejorar la infraestructura del primer cuadro, pero no vinculó a los prestadores de servicios turísticos al interior del ANP y del Corredor Ecoturístico.

Por otro lado, entre 2002 y 2014 la Conanp desarrolló en Cuatrociénegas 50 proyectos subsidiarios relacionados con la actividad turística, con inversiones del orden de los 320 mil dólares. Los proyectos incluyeron obras de infraestructura turística, capacitaciones, proyectos comunitarios y estudios de capacidad de carga turística. A pesar de este esfuerzo, la operación del Corredor Ecoturístico y el ordenamiento del turismo en el ANP no se han consolidado.

1 El progama "Pueblos Mágicos" de la Secretaría de Turismo del gobierno federal consiste en la designación de localidades que, de acuerdo con criterios determinados por esta misma Secretaría, merecen recibir un financiamiento para mejorar su aspecto en términos del paisaje urbano, principalmente, a fin de resultar más atractivos para el turismo. 


\subsection{La interpretación de los conflictos y algunas propuestas para sus soluciones}

El turismo en Cuatrociénegas comenzó a crecer lentamente tras la declaratoria del Área de Protección de Flora y Fauna, limitado desde un principio por la ausencia de infraestructura y servicios y el difícil acceso. Más tarde, las campañas de difusión y la mejora de algunas instalaciones hicieron aumentar rápidamente la afluencia de visitantes, más allá de la capacidad de carga del destino. La población local interpretó esto como un éxito económico y su percepción del turismo masivo como deseable incentivó un tipo de turismo desordenado y sin atención a la conservación del medio natural. A fin de evitar el estancamiento y la decadencia del destino turístico, la Conanp inició una nueva política orientada a modificar la percepción de la población local respecto de los efectos del turismo y promover estrategias y acciones para alcanzar un desarrollo turístico sustentable y respetuoso del ambiente, cuyos efectos a largo plazo pudieran evaluarse.

Pese a sus impactos negativos, el turismo en Cuatrociénegas continúa creciendo. Al parecer, las autoridades federales y estatales, y los propios pobladores locales, no han entendido cabalmente que la falta de control en la afluencia de turistas y el incremento no planeado de la infraestructura turística ponen en entredicho la conservación del ANP. Como se pudo observar en los resultados de las encuestas, predomina la falta de conciencia acerca de los impactos ambientales, y la mayoría de los encuestados opina que el desarrollo del turismo no afecta la conservación del ANP. Aunque los turistas tienen por lo general conocimiento de que visitan un ANP, muy pocos tienen idea de la importancia de sus recursos naturales y lo apremiante que resulta su conservación. Lo mismo ocurre con la población local, pues muy pocos residentes reconocen los servicios ambientales que derivan del sitio, lo que dificulta la implementación de los proyectos de conservación. Una manera de promover la valoración y conservación de los recursos naturales entre la población local es ampliar las oportunidades recreativas de los cieneguenses al interior del ANP.

Por otra parte, el intercambio de experiencias entre los distintos grupos e individuos que desarrollan proyectos turísticos en Cuatrociénegas permitiría aprovechar las capacidades locales existentes y vincular los servicios turísticos del Pueblo Mágico, del Corredor Ecoturístico y aquellos localizados al interior del ANP, de manera que se desarrollaran cadenas de comercio justo que redundarían en el mejoramiento y fortalecimiento de la población local. Igualmente importante es aprender de las experiencias exitosas de ecoturismo en otras ANP del país y del mundo, y promover un cambio de actitud a favor del ecoturismo.

Es importante que las autoridades de los tres órdenes de gobierno, responsables del desarrollo turístico en Cuatrociénegas se vinculen para desarrollar planes y programas con visión a largo plazo, tomando en cuenta a la población local. Es necesario reconocer que los administradores del ANP no son la única instancia responsable del desarrollo turístico a su interior y en sus alrededores. El Pueblo Mágico y el ANP conforman un binomio con grandes oportunidades para el desarrollo local y la conservación de la biodiversidad del sitio.

A pesar de los conflictos sociales entre la comunidad y la Conanp por la implementación de acciones para proteger el sitio de los embates del turismo, la población ciene- 
guense ha aceptado gradualmente la aplicación de la normatividad. Son cada vez más los prestadores de servicios turísticos que tramitan autorizaciones para desarrollar sus actividades. Para lograr que todos lo hagan es necesario combatir la impunidad y aplicar sanciones a quienes incumplan.

Las actividades recreativas y turísticas en Cuatrociénegas están íntimamente ligadas a la disponibilidad de agua en el humedal. Por ello, debe tomarse en cuenta que su conservación es la base para el desarrollo turístico del sitio. Además, el agua de las pozas es vital para el desarrollo de las actividades productivas de los cieneguenses y de todos aquellos que se benefician del agua del Valle de Cuatrociénegas fuera de la cuenca.

\section{CONCLUSIONES}

En un principio, los autores de este artículo se plantearon explorar el hecho evidente de la afectación de la biodiversidad del ANP por el desarrollo del turismo. El enfoque era principalmente cuantitativo. No obstante, la investigación fue revelando paso a paso la complejidad del fenómeno y los múltiples actores y conflictos sociales involucrados, no sólo al interior del ANP, sino también en sus alrededores. Fue así que se reconoció la necesidad de generar información que pudiera incidir a futuro en la toma de decisiones para el manejo del turismo, lo que indudablemente requería involucrar a la población local, a los inversionistas y a los distintos niveles de gobierno implicados. Esto condujo a recurrir a un enfoque cualitativo de orden constructivista que permitiera combinar y contrastar diversos puntos de vista acerca de la incidencia del turismo en un área tan frágil e importante por sus particularidades ambientales como es el ANP de Cuatrociénegas.

En el escenario de Cuatrociénegas el turismo avanza a pesar de sus impactos negativos. Muchos turistas sostienen que el turismo no afecta la conservación del ANP, y que su sola presencia es indicativa de su actitud ecológicamente responsable, aunque sus acciones no lo sean del todo. Por su parte, los cieneguenses favorecen el crecimiento del turismo, pues lo perciben como una actividad que fortalece la economía y abre oportunidades a la inversión local. No obstante, lo cierto es que el capital foráneo ha sentado sus reales en Cuatrociénegas y ha hecho cambiar la dinámica comercial hacia un ambiente de competencia desigual que desplaza a los comerciantes y negocios locales.

\section{BIBLIOGRAFÍA}

ANDERECK, K.L., VALENTINE, K.M., KNOPF, R.C., y VOGT, C.A. (2005): «Residents' Perceptions of Community Turism Impacts». Annals of Tourism Research, 32, 4: 1056-1076.

ANDRIOTIS, K., y VAUGHAN, R.D. (2003): «Urban Residents’ Attitudes Toward Tourism Development: The Case of Crete». Journal of Travel Research, 42, 2: 172-185.

AP, J. (1992): «Residents' Perceptions on Tourism Impacts». Annals of Tourism Research, 19, 4: 665-690.

ARIAS, L. M. (2009): «Interdisciplinariedad y triangulación en ciencias sociales». Diálogos revista electrónica, 10 1. Disponible en http://www.revistas.ucr.ac.cr/index.php/ dialogos/article/view/6130/5834 
ARSUAGA, G.R.B., y JIMÉNEZ, M.E.S. (2014): «Turismo justo: estrategia para combatir la pobreza en Hueyapan». El Periplo Sustentable, 26: 7-46. Disponible en http:// www.uaemex.mx/plin/psus/turismo_justo.pdf

BEZAURY-CREEL, J.E. (2009): El valor de los bienes y servicios que las áreas naturales protegidas proveen a los mexicanos. México: The Nature Conservancy/Comisión Nacional de Áreas Naturales Protegidas. Disponible en http://www.diputados.gob.mx/ documentos/noviembre/Folleto_Diputados.pdf

BRENNER, L. (2010): «Gobernanza ambiental, actores sociales y conflictos en las Áreas Naturales Protegidas mexicanas». Revista mexicana de sociología, 72, 2: 283-310. Disponible en http://www.scielo.org.mx/scielo.php?pid=S018825032010000200004yscript=sci_arttext

BRENNER,L., y JOB,H. (2006): «Actor-Management of Protected Areas and Ecotourism in Mexico». Journal of Latin American Geography, 5(2), 7-27. Disponible en: https://www. researchgate.net/profile/Ludger_Brenner/publication/236779719_Actor-Management_ of_Protected_Areas_and_Ecotourism_in_Mexico/links/54d50af50cf25013d02a661b. pdf?origin $=$ publication_list\&ev $=$ srch_pub_xdl

BRIDA, J.G., RIAÑO, E., y AGUIRRE, S.Z. (2012): «Percepciones de los residentes acerca de los impactos del turismo de cruceros en la comunidad: un análisis factorial y de clústeres». Cuadernos de turismo, (29), 79-107. Disponible en: http://revistas. um.es/turismo/article/download/153311/135191 F

CASALS, F.R., y TOURE, E.O. (2013): «El impacto del turismo en la Lengua de Barbarie (delta del río Senegal)». Cuadernos de turismo, (31), 289-309. Disponible en: http:// revistas.um.es/turismo/article/download/170881/146161

COLE, S. (2004): «Shared Benefits. Longitudinal Research in Eastern Indonesia», en PHILLMORE, J. y GOODSON, L. (Eds.). Qualitative Research in Tourism: Ontologies, Epistemologies and Methodologies. New York: Routledge, pp. 292-310.

COLE, S. (2005): «Action Ethnography: Using Participation Observation», en RITCHIE, B., BURNS, P., y PALMER, C. (Eds.). Tourism Research Methods: Integrating Theory with Practice. Cambridge, MA: CAB International, pp. 63-72. Disponible en https:// books.google.com.mx/books?id=pd1SD7_8_YsC\&pg=PA63\&dq=Action+Ethnograph $\mathrm{y}:+$ Using+Participation+Observation+cole\&hl=en\&sa=X\&ei=Ec-UVYnKH4bLsAX TwJPIBw\&ved=0CB4Q6AEwAA\#v=onepage \&q=Action $\% 20$ Ethnography $\% 3 \mathrm{~A} \% 20$ Using\%20Participation\%20Observation \%20 cole \&f=false

COLE, STROMA (2007): «Entrepreneurship and Empowerment: Considering The Barriers - A Case Study from Indonesia». Turizam: znanstveno-stručni časopis, 55, 4: 461-473. Disponible en: http://hrcak.srce.hr/24675

COMITÉ PUEBLO MÁGICO (2011): Propuesta Cuatrociénegas Pueblo Mágico. Cuatrociénegas, Coahuila, México: Comité Propueblo Mágico.

CONABIO (2009): «Capital natural de México» vol. II: Estado de conservación y tendencias de cambio. México: Comisión Nacional para el Conocimiento y Uso de la Biodiversidad.

CONAGUA (2009): Manifestación de impacto ambiental. Modalidad regional del proyecto Modernización del Riego del Valle de Cuatrociénegas, Coahuila. Saltillo, México: Comisión Nacional del Agua. 
CONANP (2007a): Programa de Turismo en Áreas Protegidas 2007-2012. México: Comisión Nacional de Áreas Naturales Protegidas/Secretaría de Medio Ambiente y Recursos Naturales. Disponible en http://www.conanp.gob.mx/pdf_publicaciones/ Turismopags-individuales.pdf

CONANP (2007b): Estudio sobre el cambio de uso de suelos en el Valle de Cuatrociénegas, periodo 2002-2006. Morelia, México: Comisión Nacional de Áreas Naturales Protegidas. Disponible en http://cuatrocienegas.conanp.gob.mx/docs/Tasa2006.pdf

CONANP (2008a): Programa del Operativo Semana Santa 2008 para el Área de Protección de Flora y Fauna Cuatrociénegas. México: Secretaría de Medio Ambiente y Recursos Naturales/Comisión Nacional de Áreas Naturales Protegidas.

CONANP (2008b): Resumen de acciones del Operativo Semana Santa 2008 para el Área de Protección de Flora y Fauna Cuatrociénegas. México: Secretaría de Medio Ambiente y Recursos Naturales/Comisión Nacional de Áreas Naturales Protegidas.

CONANP (2008c): Información turística del Área de Protección de Flora y Fauna Cuatrociénegas. México: Secretaría de Medio Ambiente y Recursos Naturales/ Comisión Nacional de Áreas Naturales Protegidas.

CONANP (2012): Nivel de conocimiento de los objetos de conservación y sus amenazas en el Área de Protección de Flora y Fauna Cuatrociénegas. Reporte 2012. México: Comisión Nacional de Áreas Naturales Protegidas.

CONANP (2014): Programa Nacional de Áreas Naturales Protegidas 2014-2018. México: Comisión Nacional de Áreas Naturales Protegidas. Disponible en http://entorno. conanp.gob.mx/documentos/PNANP.pdf

DE OLIVEIRA, J.A.P. (2002): «Implementing Environmental Policies in Developing Countries Through Decentralization: The Case of Protected Areas in Bahia, Brazil». World Development, 30, 10: 1713-1736.

DECROP, A. (2004): «Trustworthiness in Qualitative Tourism Research», en PHILLMORE, J. y GOODSON, L. Qualitative Research in Tourism. Ontologies, Epistemologies and Methodologies. USA: Routledge, pp. 156-169.

ESCALANTE, A.E., EGUIARTE, L.E., ESPINOSA-ASUAR, L., FORNEY, L.J., NOGUEZ, A.M., y SALDÍVAR, V.S. (2008): «Diversity of Aquatic Prokaryotic Communities in the Cuatro Cienegas Basin». FEMS Microbiology Ecology, 65,1: 50-60. Disponible en http://femsec.oxfordjournals.org/content/65/1/50.abstract

GALVÁN, D. (2007): Y nosotros ¿qué? Incorporación del Ejido La Vega al Área de Protección de Flora y Fauna de Cuatrociénegas. Tesis de licenciatura en Antropología. Puebla, México: Escuela de Ciencias Sociales, Artes y Humanidades, Universidad de las Américas. Disponible en http://catarina.udlap.mx/u_dl_a/tales/documentos/laac/ galvan_b_d/indice.html

GARCÍA, E. (1987): Modificaciones al sistema de clasificación climática de Köppen. Cuarta Edición. México: Universidad Nacional Autónoma de México.

GARCÍA, I., OLIVERA, D., PEDROZA, A., MARTÍNEZ, J.G. y CUETO, J.A. (2006): «Recreación y sus impactos en la Reserva de la Biosfera Mapimí». Revista Chapingo. Serie Zonas Áridas. 5, 2:151-161. Disponible en http://www.chapingo.uruza.edu.mx/ images/revista/vol5-num2-2006.pdf 
GRALL, G. (1995): «Cuatrociénegas: México’s Desert Aquarium». National Geographic, 188 (Octubre): 85-97.

INAPAC (2014): Diágnosticos municipales del Programa de Apoyo a la Comunidad y Medio Ambiente: Cuatrociénegas. México: Instituto Nacional de la Administración Pública A.C./Petróleos Mexicanos. Disponible en https://pacma.org.mx/solicitudes/ files/diagnostico/Diagnostico_05007_Cuatro_Cienegas_Coah.pdf

INE (1999): Programa de Manejo del Área de Protección de Flora y Fauna Cuantrociénegas. México: Instituto Nacional de Ecología. Disponible en http://www.inecc.gob. $\mathrm{mx} /$ descargas/publicaciones/180.pdf

INEGI (2010): Censo de Población y Vivienda 2010. Resultados preliminares, Instituto Nacional de Estadística y Geografía, Aguascalientes, México.

INEGI (2011): Panorama sociodemográfico de Coahuila de Zaragoza. Aguascalientes, México: Instituto Nacional de Estadística y Geografía. Disponible en http://www. inegi.org.mx/prod_serv/contenidos/espanol/bvinegi/productos/censos/poblacion/2010/ panora_socio/coah/Panorama_Coah.pdf

INEGI (2013): Marco Geoestadístico Municipal 2013 Versión 6.0 (Inventario Nacional de Viviendas 2012), Instituto Nacional de Geografía y Estadística, Aguascalientes, México.

INEGI (2015): Estadísticas a propósito del día internacional del turismo 2015. Instituto Nacional de Estadística y Geografía. Aguascalientes, México. 11 pp. Disponible en http://www.inegi.org.mx/saladeprensa/aproposito/2015/turismo0.pdf

JUROWSKI, C., y GURSOY, D. (2004): «Distance Effects on Residents’ Attitudes Toward Tourism». Annals of Tourism Research, 31, 2: 296-312.

LOBO, T. (2009): Cuatrociénegas: La mirada de sus niños. México: Proyecto Concentrarte/World Wildlife Fund/Fundación Carlos Slim.

LÓPEZ, A. GARCÍA, I., SALAS, C. (2009): «Turismo en la Reserva de la Biosfera Mapimí», en LÓPEZ, A. y SÁNCHEZ A. (Eds.). Comarca Lagunera: procesos territoriales regionales en el contexto global. México: Instituto de Geografía, UNAM, pp. (413-436).

MAEKAWA, M., LANJOUW, A., RUTAGARAMA, E., y SHARP, D. (2013): «Mountain Gorilla Tourism Generating Wealth and Peace in Post-Conflict Rwanda». Natural Resources Forum, 37, 2: 127-137.

MÁRQUEZ, A.R. y SÁNCHEZ, A. (2007): «Turismo y ambiente: la percepción de los turistas nacionales en Bahía de Banderas, Nayarit, México». Investigaciones Geográficas, Boletín del Instituto de Geografía, UNAM. 64: 34-152. Disponible en http://www. scielo.org.mx/scielo.php?pid=S0188-46112007000300009yscript=sci_arttextytlng=pt

MARTIN, D.G. (2010): «Reflections on Teaching Qualitative Methods in Geography», en DeLyser, D., HERBERT S., AITKEN, S., CRANG, M., y MCDOWELL, L. (Eds.). The Sage Handbook of Qualitative Geography. London: SAGE Publications Ltd., pp. 406-417.

MEIXUEIRO, G.M. (2008): Impacto de la actividad turística en el desarrollo local. México: Centro de Estudios Sociales y Opinión Pública. Documento de trabajo número 48. Disponible en http://www3.diputados.gob.mx/camara/content/download/193968/465270/file/Iimpacto_por ciento20turismo_desarrollo_docto48.pdf 
MEYER, E. R. (1973): «Late-Quarternary Paleoecology of the Cuatro Cienegas Basin, Coahuila, Mexico». Ecology (54)5: 982-995. Disponible en http://www.jstor.org/ stable/1935565?seq=1\#page_scan_tab_contents

MONTERRUBiO, J. C., Mendoza, M. M., FERnÁNDEZ, M. J., y GULlETE, G. S. (2011): «Turismo y cambios sociales. Estudio cualitativo sobre percepciones comunitarias en bahías de Huatulco, México». Cuadernos de turismo, (28), 171-189. Disponible en: http://revistas.um.es/turismo/article/download/147291/131331

MONTERRUBIO, J.C., MENDOZA, M.M. y HUITRÓN, T.K. (2013): «Percepción de la comunidad local sobre los impactos sociales del 'spring break' en Acapulco, México». El Periplo Sustentable, 24: 41-65. Disponible en http://moodle2.unid.edu.mx/dts_cursos_mdl/lic/AET/TR/AM/7y8/Percepciones.pdf

NÚÑEZ, J.J. (2006): Analysis and Proposal of a Sustainable Development Strategy for the Valley of Cuatrociénegas, Based on Principles of Urban Planning and Urban Governance. Single Case Study. Estocolmo: Royal Institute of Technology. Department of Urban Planning and the Environment. Division of Built Environment Analysis. Disponible en https://www.kth.se/polopoly_fs/1.190792!/Menu/general/column-content/ attachment/MScThesNunez06.pdf

NUNKOO, R., y GURSOY, D. (2012): «Residents' Support for Tourism: An Identity Perspective». Annals of Tourism Research, 39, 1: 243-268.

ORGANIZACIÓN INTERNACIONAL DEL TRABAJO (2011): Guía práctica sobre la reducción de la pobreza a través del turismo. Ginebra: OIT.

PÉREZ, C., CRUZ, G., y CAMACHO, A. (2010): «Análisis del aprovechamiento turístico del parque estatal Sierra de Tepotzotlán, México desde la perspectiva de la sustentabilidad». El Periplo Sustentable, (19), 35-68. Disponible en http://rperiplo.uaemex.mx/ index.php/elperiplo/article/viewFile/908/661

PHILLMORE, J. y GOODSON, L. (2004): «Progress in Qualitative Research in Tourism: Epistemology, Ontology and Methodology», en Qualitative Research in Tourism. Ontologies, Epistemologies and Metodologies. New York: Routledge, pp. 2-29.

ROE, D., MOHAMMED, E. Y., PORRAS, I., y GIULIANI, A. (2013): «Linking Biodiversity Conservation and Poverty Reduction: Depolarizing the Conservation-Poverty Debate. Conservation Letters, 6, 3; 162-171. Disponible en http://onlinelibrary.wiley. com/doi/10.1111/j.1755-263X.2012.00292.x/full

SALAS, A.I., ALONSO, F. y CASTAÑEDA, G. (2010): Informe de actividades para el monitoreo de tortuga de Cuatrociénegas (Terrapene coahuila) en el Área de Protección de Flora y Fauna Cuatrociénegas. Gómez Palacio, México: Universidad Juárez del Estado de Durango.

SECRETARÍA DE GOBERNACIÓN (1994): «Decreto por el que se declara como área natural protegida, con el carácter de área de protección de flora y fauna, la región conocida como Cuatrociénegas, con una superficie de 84,347-47-00 hectáreas, municipio del mismo nombre, Coahuila». México: Diario Oficial de la Federación. Disponible en http://www.conanp.gob.mx/sig/decretos/apff/Cuatrocienegas.pdf

SECRETARÍA DE GOBERNACIÓN (2008): «Acuerdo por el que se dan a conocer los estudios técnicos del Acuífero 0528 Cuatrociénegas y se modifican los límites y planos de localización que respecto del mismo se dieron a conocer en el Acuerdo por el que 
se dan a conocer los límites de 188 acuíferos de los Estados Unidos Mexicanos, los resultados de los estudios realizados para determinar su disponibilidad media anual de agua y sus planos de localización». México: Diario Oficial de la Federación. Disponible en: http://dof.gob.mx/nota_detalle_popup.php?codigo=5045495

SECRETARÍA DE GOBERNACIÓN (2013): «Decreto por el que se establece como zona reglamentada aquella que ocupa el acuífero denominado Cuatrociénegas, ubicado en el Estado de Coahuila». México: Diario Oficial de la Federación. Disponible en: http:// www.dof.gob.mx/nota_detalle.php?codigo $=5312514 \mathrm{yfecha}=02 / 09 / 2013$

SECTUR (2007): Elementos para evaluar el impacto económico, social y ambiental del turismo de naturaleza en México. México: Secretaría de Turismo. Disponible en http://ictur.sectur.gob.mx/pdf/estudioseinvestigacion/sustentabilidad/METODOLOGIA_SUSTENTABILIDAD_1d3.pdf

SISTEMA INTEGRAL DE INFORMACIÓN DE MERCADOS TURÍSTICOS (2015): Situación del sector turístico: 2014 y avances 2015. México: Secretaría de Turismo/ Consejo Nacional de Promoción Turística. Disponible en http://www.siimt.com/es/ siimt/siim_inicio

SOLARI, A. y PÉREZ, M. (2005): «Desarrollo local y turismo: relaciones, desavenencias y enfoques». Economía y sociedad. Universidad Michoacana de San Nicolás de Hidalgo. México, 10, 16: 49-46.

SOUZA, V., ESPINOSA-ASUAR, L., ESCALANTE, A.E., EGUIARTE, L.E., FARMER, J., FORNEY, L. y ELSER, J.J. (2006): «An Endangered Oasis of Aquatic Microbial Biodiversity in the Chihuahuan Desert». Proceedings of the National Academy of Sciences, 103, 17: 6565-6570. Disponible en http://www.pnas.org/content/103/17/6565

SUSANA, S.P. (2005): «Analisis de la actividad turística en Áreas Naturales Protegidas. México»: CIIEMAD-IPN. (112). Disponible en http://itzamna.bnct.ipn.mx/dspace/bitstream/123456789/1413/1/427_2005_CIIEMAD_MAESTRIA_susana_bugueno.pdf

TINOCO, G. (2003): «Los impactos del turismo en el Perú». Industrial Data. Lima: Universidad Nacional Mayor de San Marcos, 6, 1: 47-60. Disponible en http://revistasinvestigacion.unmsm.edu.pe/index.php/idata/article/view/5982/5179

WANG, Y., PFISTER, R.E., y MORAIS, D.B. (2006): Residents' Attitudes toward tourism development: A case study of Washington, NC, en Proceedings of the Northeastern Recreation Research Symposium, pp. 411-418. Disponible en: http://www.nrs.fs.fed. us/pubs/gtr/gtr_nrs-p-14/54-wang-p-14.pdf

WATSON, J.E., DUDLEY, N., SEGAN, D.B., y HOCKINGS, M. (2014): «The Performance and Potential of Protected Areas». Nature, 515, 7525: 67-73. Disponible en: http://www.nature.com/nature/journal/v515/n7525/full/nature13947.html

YEASMIN, S., y RAHMAN, K.F. (2012): «Triangulation Research Method as the Tool of Social Science Research». Bup Journal, 1, 1: 154-163. Disponible en: http://www. bup.edu.bd/journal/154-163.pdf 